\title{
Solitary Necrotic Nodule of Liver with the Etiology of Parasite: A Report of Two Cases
}

\author{
Mingwei Sui, MD ${ }^{a}$, Wenzhao Liang, MD ${ }^{b}$, Ting Zhang, MD ${ }^{a}$, Yang Bai, MD ${ }^{a}$, Dezhi Zhang, MD ${ }^{a, *}$ \\ ${ }^{a}$ Department of Abdomen Ultrasound, First Hospital of Jilin University, 71 Xinmin Road, Changchun, 130021, China; ${ }^{b}$ Department of \\ Neurology, China-Japan Union Hospital of Jilin University, 126 Xiantai Street, Changchun, 130021, China. \\ Received November 12, 2018; revision requested February 12, 2019; revision received March 1; accepted March 8.
}

\begin{abstract}
Solitary necrotic nodule of liver (SNNL) is a rare, benign lesion with an uncertain etiology. There are no typical diagnostic clinical or radiological features. The two patients we described underwent liver resection because it was not possible to exclude a malignancy on preoperative imaging. The histopathological findings suggest the SNNL may be parasitic in origin and find parasitic worm in the nodules.
\end{abstract}

Key words: Solitary necrotic nodule (SNN); Parasite; Liver

Advanced Ultrasound in Diagnosis and Therapy 2019;01:018-021

DOI: 10.37015/AUDT.2019.190804

\section{Case 1}

A 60-year-old female came to our hospital for blood in the faeces of one month. Physical examination showed no obvious abnormality. The blood test was normal. An abdominal ultrasound displayed a nodular formation in the right lobe of the liver. The contrastenhanced ultrasound exposed a peripheral enhancement in the arterial phase and low contrast enhancement in delay phase suggestive of metastasis (Fig. 1). Colorectal computed tomography (CT) with contrast suggestive of rectum carcinoma and a low contrast enhancement nodule in posterior segment of right lobe in delay phase (Fig. 2). She underwent open radical resection and anatomical VII segmentectomy of the liver. After surgery, there were five nodular mass in liver specimen, gray, materiality and tough. With hematoxylin-eosin staining the specimen, there were multiple granulomatous necrosis, with parasitic worm in it (Fig. 3), suggestive of parasitic necrotic nodules.

\section{Case 2}

A 41-year-old female came to our hospital for focal liver lesions of two weeks, who has a family history of hepatitis B. Physical examination showed right upper quadrant tenderness. An abdominal ultrasound showed a nodule in the right lobe of the liver (Fig. 4). The enhanced MRI indicated a nodule in posterior segment of right lobe with no significant enhancement (Fig. 5). She underwent laparoscopic anatomical VII segmentectomy of the liver. During the procedure, two adjacent, rough, gray nodules were found in the posterior segment of the right lobe liver, near the top of the diaphragm. Hematoxylin-eosin staining the specimen reported a solitary necrotic nodule of liver, with parasitic worm in it (Fig. 6).

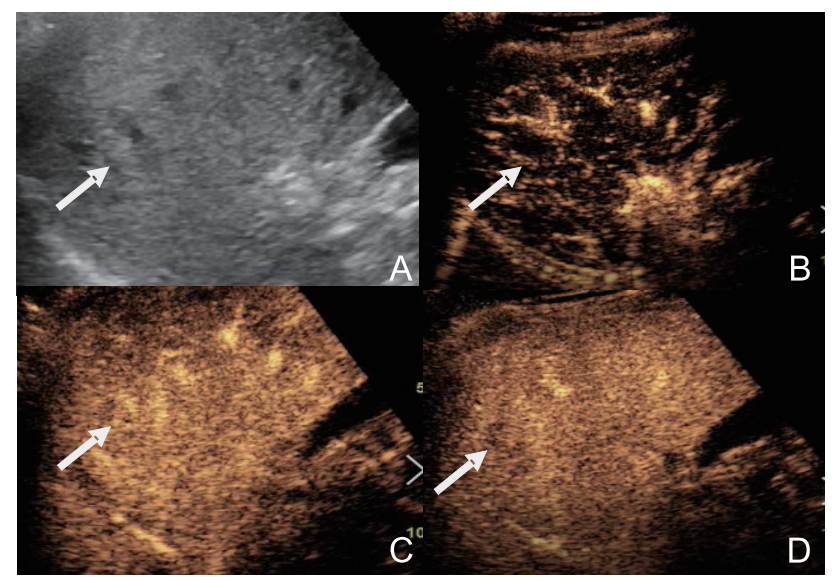

Figure 1 (A) Abdominal ultrasound show a nodule in posterior segment of right lobe, $8 \times 7 \mathrm{~mm}$. (B) Peripheral enhancement in the arterial phase of contrast-enhanced ultrasound. (C) Low contrast enhancement in portal venous phase of contrast-enhanced ultrasound. (D) Low contrast enhancement in delay phase of contrast-enhanced ultrasound.

\footnotetext{
* Corresponding Author: Department of Abdominal Ultrasound, 1st Hospital of Jilin University, Changchun, Jilin Province 130021, China

e-mail: zhangdezhi1982@hotmail.com
} 

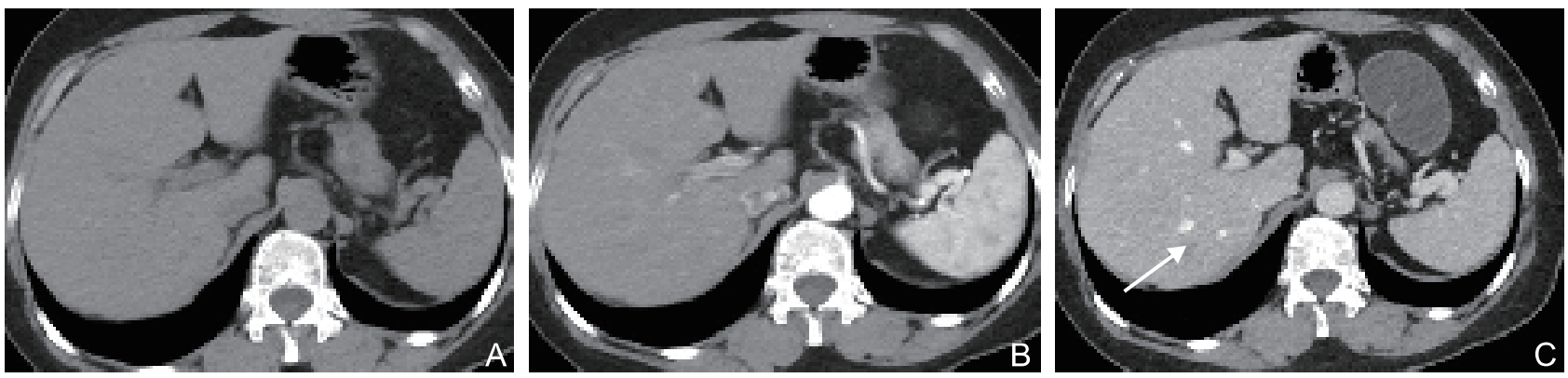

Figure 2 (A) Hepatic nodule is not visible in CT scan. (B) Hepatic nodule is not visible in CT scan arterial phase. (C) A nodule in posterior segment of right lobe, $6 \times 7 \mathrm{~mm}$, low contrast enhancement in delay phase of contrast-enhanced CT.

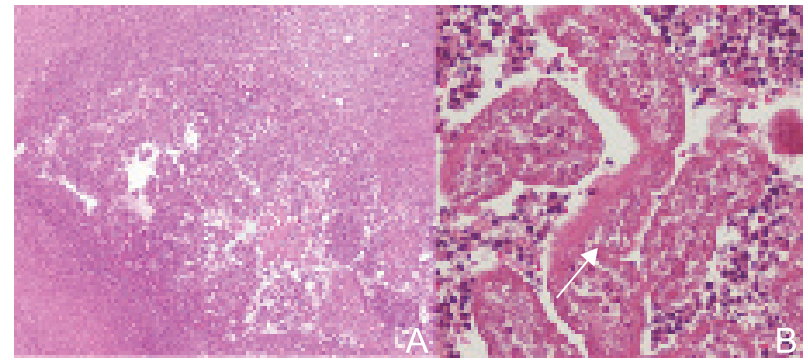

Figure 3 (A) Multiple granulomatous necrosis accompanied by a large number of inflammatory cells infiltration $(\mathrm{H} \& \mathrm{E}, \times 40)$. (B) Multiple damaged parasitic worms in centre $(\mathrm{H} \& \mathrm{E}, \times 400)$.

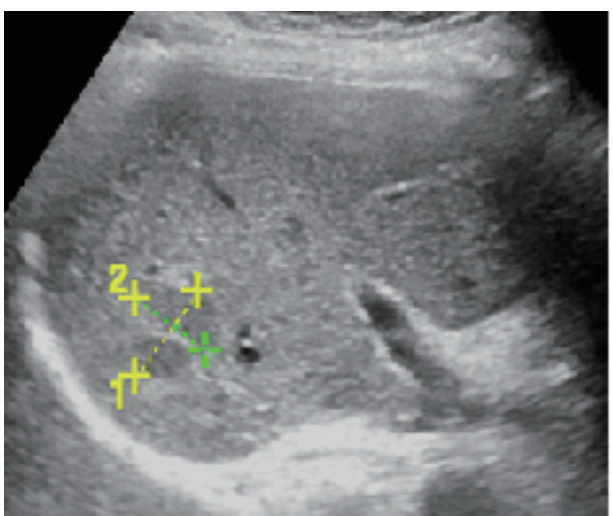

Figure 4 (A) nodule in posterior segment of right lobe, $24 \times 16 \mathrm{~mm}$, boundary is clear, echo is uneven.
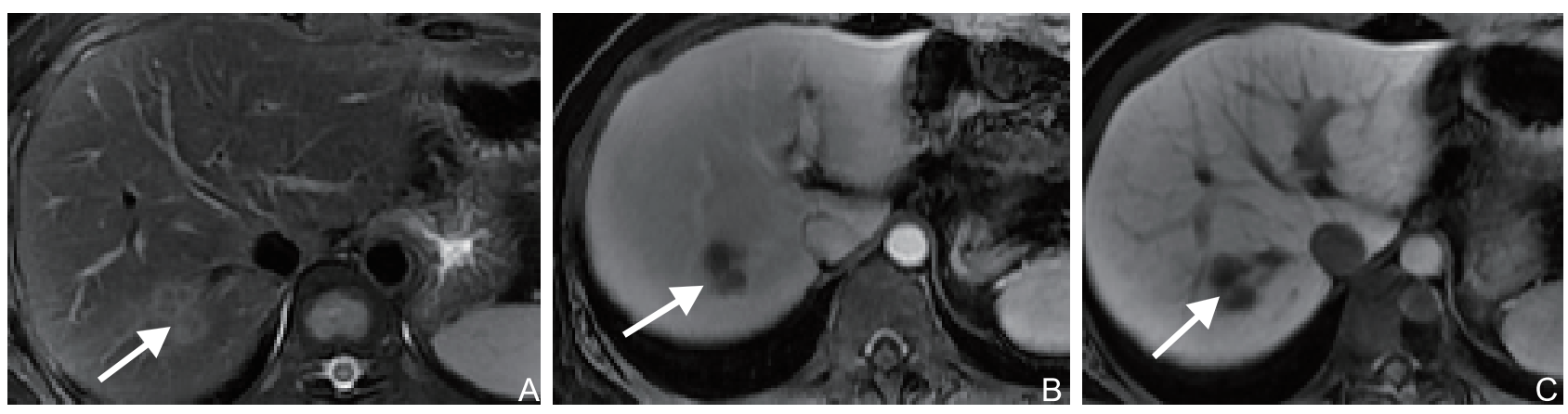

Figure 5 Enhanced MRI suggestive of a nodule in posterior segment of right lobe, $24 \mathrm{~mm} \times 25 \mathrm{~mm}$, with no significant enhancement plain scan (A), arterial phase (B), venous phase (C)).
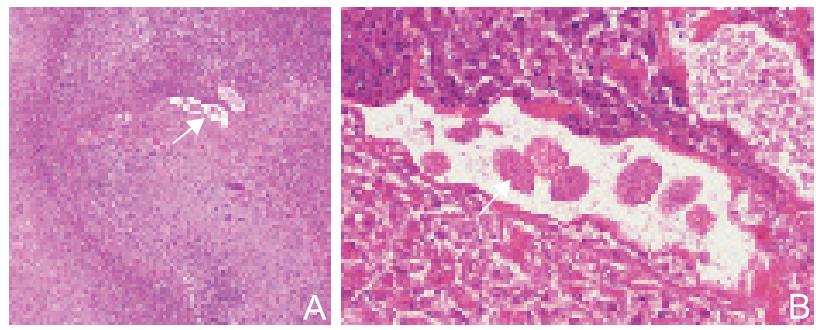

Figure 6 (A) The centre of the coagulative necrosis, with a fibrous capsule containing inflammatory cells $(\mathrm{H} \& \mathrm{E}, \times 40)$. (B) Multiple damaged parasitic worms in centre $(\mathrm{H} \& \mathrm{E}, \times 400)$.

The patient was discharged on postoperative day 4 , without any complications. The patient remains asymptomatic without signs of relapse of SNNL to date.

\section{Discussion}

Solitary necrotic nodule (SNN) is an uncommon hepatic lesion, pathologically characterized by central necrotic core encapsulated with a hyalinized fibrotic tissue containing elastic fibers and inflammatory cells [1-4]. SNNL is a rare benign lesion with an uncertain etiology. The etiology and pathogenesis of the lesion still remain ambiguous. However, it has been suggested by various researchers that it is related to infections like tuberculosis and syphilis, parasitic infestations, trauma and sclerosing haemangiomas [2,5]. Possibility of 
SNNL arising from two or more different mechanisms simultaneously has also been suggested [6].

Furthermore, many patients with SNN show the tendency to be accompanied by primary cancer of other organs $[7,8]$. Many studies have emphasized that these lesions may be erroneously diagnosed as metastatic deposits, both clinically and radiologically $[5,6]$. In a retrospective study including 22 cases, SNNL was frequently found in patients with liver metastases, Deniz et al. speculated that at least some of these lesions may represent 'burnt out' tumours [9]. Undoubtedly, some patients experience spontaneous regression of liver tumours or have an excellent response to chemotherapy, this may explain some lesions that are reported as SNNL. The first case we described had rectum carcinoma, so we highly suspect that her liver nodule was metastatic at the beginning.

The most plausible etiology is that these lesions are the result of an immune response to infection, particularly a parasitic infection. In case of parasitic origin, degenerated larva can be seen in the necrotic center as in our cases [10]. Some specific larvae, Clonorchis sinesis [5], filarial nematode [11], Capillaria hepatica [12], and hydatid cyst [9] had been detected in SNN. However, the larval structure is usually over degraded, so it is difficult to identify the exact species. In recent years, changes in dietary lead to more parasitic infection, which mainly affects the gastrointestinal tract and biliary tract. Moreover, other organs infections have been found due to the advances of imaging modalities. The liver, as the main organ of the digestive system, is often infected with parasites. Many foodborne parasites can cause liver infections. Tsui et al. discovered clonorchis sinensis in two of seven patients with SNNL [5]. More recently, a case described by Koea et al. was attributed to capillaria hepatica infection [13]. There are still some rare parasitic infections, such as pinworm, etc. There is a case of SNN with etiology of pinworm which is one of subspecies of nematodes. Liver pinworm infection was extremely rare [14]. Rarely, Pinworm is found outside of the gastrointestinal tract in the peritoneum, fallopian tubes and liver [14-16]. It is suggested that a breach in intestinal continuity permits translocation of the parasite out of the intestine. The patient of our first case had rectum carcinoma, maybe the carcinoma gave an opportunity for pinworm transferring to liver.

We were familiar with liver hydatid disease, liver schistosomiasis previously. However, , the nodules did not meet the typical imaging feature of liver hydatid disease or liver schistosomiasis in the two cases we described. Since we suspected the liver nodules were metastatic cancer initially without considering to be parasitic nodules, so we ruled out to check the patients' stool to find worm ova which is the simplest and most accurate diagnostic method. Parasitic worm in pathological specimen was detected ultimately.

SNNL imaging features is challenging and requires a combination of trans-abdominal US, multiphase CT scanning and an MRI scanning generally. Unfortunately, no single radiological technique can confidently diagnose SNNL [17]. With regard to CT imaging, Yoon et al. concluded that it was difficult, if not impossible, to differentiate SNNL from hepatic metastases using this modality alone [18]. At present, MRI is believed to be specific for its diagnosis. In most cases, there was no internal enhancement in arterial phase, portal vein phase and delayed phase, and some edge enhancement was observed, which was more obvious in delayed phase images $[8,12]$. The combination of clinical and imaging examination is conducive to the accurate diagnosis of preoperative SNNL. However, clinical data and imaging features of many cases are not typical, and may suggest the possibility of other diseases. This eventually led to misdiagnosis and unnecessary hepatectomy, like our second case.

\section{Conclusion}

Since imaging diagnosis is often controversial, maybe we can make a diagnosis from the etiology. Parasite is an important etiology of SNNL, more and more SNNL caused by parasites have been diagnosed. So stool microbial examination will be helpful in patients with an ambiguous diagnosis, and should be normalized, in order to minimize unnecessary hepatectomy.

\section{Conflicts of interest}

This work was supported by the National Natural Science Foundation of China (Grant No. 81501478), the National Science and Technology Major Project of China (2017ZX10203204-008-002) and the Jilin Scientific and Technological Development Program (20180520119JH). The funders had no role in study design, data collection and analysis, decision to publication, or preparation of the paper.

\section{References}

[1] Geng L, Lin C, Huang B, Long XA, Dai BH, Cong WM, et al. Solitary necrotic nodule of the liver: MR findings in 33 pathologically proved lesions. Eur J Radiol 2012;4:623-9.

[2] Zhou YM, Li B, Xu F, Wang B, Li DQ, Liu P, et al. Clinical features of solitary necrotic nodule of the liver. Hepatobiliary Pancreat Dis Int 2008;5:485-9.

[3] Sundaresan M, Lyons B, Akosa AB. 'Solitary' necrotic nodules of the liver: an aetiology reaffirmed. Gut 1991;11:1378-80.

[4] Shepherd NA, Lee G. Solitary necrotic nodules of the liver simulating hepatic metastases. J Clin Pathol 1983;10:1181-3.

[5] Tsui WM, Yuen RW, Chow LT, Tse CC. Solitary necrotic nodule of the liver: parasitic origin? J Clin Pathol 1992;45(11):975-8. 
[6] Patti R, Cabibi D, Sparacello M, Di Vita G, Montalto G. Solitary necrotic nodule of the liver: different pathological findings express a different histogenesis. Case Rep Gastroenterol 2008;1:149-54.

[7] Deniz K, Artış T, Tekelioğlu F, Özcan S. Solitary necrotic nodule of the liver. J Gastrointestin Liver Dis. 2011;20(4):346.

[8] Iwase K, Higaki J, Yoon HE, Mikata S, Miyazaki M, Torikai K, et al. Solitary necrotic nodule of the liver. J Hepatobiliary Pancreat Surg 2002;1:120-4.

[9] Deniz K, Coban G. Solitary necrotic nodule of the liver: always benign? J Gastrointest Surg 2010;3:536-40.

[10] Noh SJ, Jachin S, Moon WS. Solitary necrotic nodule of the liver. Clin Mol Hepatol. 2012;2:235-8.

[11] Clouston AD, Walker NI, Prociv P. Parasitic origin of a solitary necrotic nodule of the liver. J Clin Pathol.1993;6:578.

[12] Koea J, Taylor G, Miller M, Rodgers M, McCall J. Solitary necrotic nodule of the liver: a riddle that is difficult to answer. $J$ Gastrointest Surg 2003;5:627-30.
[13] Koea J, Smith D. Solitary necrotic nodule revisited. ANZ J Surg. 2008;1-2:109-11.

[14] Ng WS, Gallagher J, McCaughan G. "Pinworm" infection of the liver: unusual CT appearance leading to hepatic resection. Dig Dis Sci 2004;3:466-8.

[15] MacPherson D. Intestinal parasites in returned travelers. Med Clin North Am. 1999;4:1053-75.

[16] Saxena AK, Springer A, Tsokas J, Willital GH. Laparoscopic appendectomy in children with Enterobius vermicularis. Surg Laparosc Endosc Percutan Tech. 2001;4:284-6.

[17] Pananwala H, Pang TC, Eckstein RP, Hudson BJ, Newey A, Samra JS, et al. The enigma of solitary necrotic nodule of the liver. ANZ J Surg 2014;4:260-5.

[18] Yoon K, Yun K, Lee J, Kim C. Solitary necrotic nodules of the liver mimicking hepatic metastasis: report of two cases. Korean J Radiol. 2000;1(3):165-8. 